\title{
Developmental exposure to the DE-71 mixture of polybrominated diphenyl ether (PBDE) flame retardants induce a complex pattern of endocrine disrupting effects in rats
}

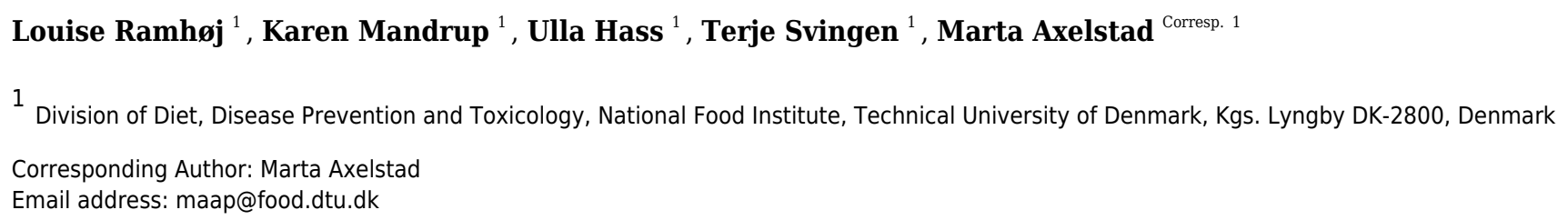

Polybrominated diphenyl ethers (PBDEs) are legacy compounds with continued widespread human exposure. Despite this, developmental toxicity studies of DE-71, a mixture of PBDEs, are scarce and its potential for endocrine disrupting effects in vivo is not well covered. To address this knowledge gap, we carried out a developmental exposure study with DE-71. Pregnant Wistar rat dams were exposed to 0,40 or $60 \mathrm{mg} / \mathrm{kg}$ bodyweight/day from gestation day 7 to postnatal day 16 , and both sexes were examined. Developmental exposure affected a range of reproductive toxicity endpoints. Effects were seen for both male and female anogenital distances (AGD), with exposed offspring of either sex displaying around $10 \%$ shorter AGD compared to controls. Both absolute and relative prostate weights were markedly reduced in exposed male offspring, with about $40 \%$ relative to controls. DE-71 reduced mammary gland outgrowth, especially in male offspring. These developmental in vivo effects suggest a complex effect pattern involving anti-androgenic, anti-estrogenic and maybe estrogenic mechanisms depending on tissues and developmental stages. Irrespective of the specific underlying mechanisms, these in vivo results corroborate that DE-71 causes endocrine disrupting effects and raises concern for the effects of PBDE-exposure on human reproductive health, including any potential long-term consequences of disrupted mammary gland development. 
1 Developmental exposure to the DE-71 mixture of polybrominated diphenyl

2 ether (PBDE) flame retardants induce a complex pattern of endocrine

3 disrupting effects in rats

4

5 Louise Ramhøj ${ }^{1}$, Karen Mandrup ${ }^{1}$, Ulla Hass ${ }^{1}$, Terje Svingen ${ }^{1}$ and Marta Axelstad ${ }^{1}$

6

7 'Division of Diet, Disease Prevention and Toxicology, National Food Institute, Technical University of

8 Denmark, Kgs. Lyngby DK-2800, Denmark

10 Corresponding author:

11 Marta Axelstad ${ }^{1}$

12 Kemitorvet, Bygning 202, rum 5211, DK-2800 Kgs. Lyngby, Denmark

13 Tel: +4535887544

14 Email: maap@food.dtu.dk

15 Running Title: Endocrine disrupting effects of DE-71

16 Key words: mammary gland, anti-androgenic, flame retardants, polybrominated diphenyl ethers,

17 development, endocrine disruption, anogenital distance, nipple retention 


\section{ABSTRACT}

19 Polybrominated diphenyl ethers (PBDEs) are legacy compounds with continued widespread

20 human exposure. Despite this, developmental toxicity studies of DE-71, a mixture of PBDEs, are

21 scarce and its potential for endocrine disrupting effects in vivo is not well covered. To address

22 this knowledge gap, we carried out a developmental exposure study with DE-71. Pregnant

23 Wistar rat dams were exposed to 0,40 or $60 \mathrm{mg} / \mathrm{kg}$ bodyweight/day from gestation day 7 to

24 postnatal day 16, and both sexes were examined. Developmental exposure affected a range of

25 reproductive toxicity endpoints. Effects were seen for both male and female anogenital distances

26 (AGD), with exposed offspring of either sex displaying around 10\% shorter AGD compared to

27 controls. Both absolute and relative prostate weights were markedly reduced in exposed male

28 offspring, with about $40 \%$ relative to controls. DE-71 reduced mammary gland outgrowth,

29 especially in male offspring. These developmental in vivo effects suggest a complex effect

30 pattern involving anti-androgenic, anti-estrogenic and maybe estrogenic mechanisms depending

31 on tissues and developmental stages. Irrespective of the specific underlying mechanisms, these in

32 vivo results corroborate that DE-71 causes endocrine disrupting effects and raises concern for the

33 effects of PBDE-exposure on human reproductive health, including any potential long-term

34 consequences of disrupted mammary gland development. 


\section{Introduction}

36 Normal development through gestation and early postnatal life is essential for lifelong health and

37 disruption to developmental processes can result in adverse effects and increased susceptibility to

38 disease. Since the environment where development occurs plays a key role in ensuring proper

39 development, environmental stressors are also major contributors to the development of diseases.

40 This includes endocrine disrupting chemicals, which can disturb molecular and biological

41 pathways that, through their effects on the endocrine systems, can change developmental

42 processes and cause adverse health effects (Gore et al., 2015).

43 Polybrominated diphenyl ethers (PBDEs) are legacy compounds that were widely used as flame

44 retardants in various consumer and industrial products. They have been shown to cause

45 numerous adverse health effects in humans and animals and are now restricted under the

46 Stockholm Convention on Persistent Organic Pollutants (Stockholm Convention, 2019).

47 Unfortunately, humans are still exposed to PBDEs, as they are persistent in indoor and outdoor

48 environments and bioaccumulate.

Among the many human health effects for which PBDEs have been implicated, thyroid hormone

50 system disruption, neurotoxicity and reproductive disorders are prevalent (Boas, Feldt-

51 Rasmussen \& Main, 2012; Attina et al., 2016; Trasande et al., 2016). Still, their specific

52 endocrine mechanisms of action are not well understood and a characterization of the adverse

53 reproductive effects in perinatally exposed rat offspring remain lacking. Even though PBDEs are

54 largely banned, such information is still valuable as human exposure will continue and since it

55 will help us better understand the current disease burden of these legacy chemical substances as

56 well as of endocrine disruptors in general. Perhaps equally important is its applicability towards

57 improvement of current safety assessment regimens, including considerations for mixture effects. 
58 Also experimental studies have shown various potential endocrine effects of DE-71 and its

59 metabolites. Amongst the effects potentially relevant for reproductive health are weak anti-

60 estrogenic and estrogenic effects in vitro and in vivo (Mercado-Feliciano \& Bigsby, 2008a,b) as

61 well as clear anti-androgenic properties in vitro (Stoker et al., 2005) and in exposed male rats

62 (Stoker et al., 2004, 2005), while the potential effects of DE-71 in a developmental toxicity

63 scenario are understudied. A recent meta-analysis on PBDEs suggest broad adverse reproductive

64 effects in postnatal male rats (Zhang et al., 2020). Based on this, we surmised that the DE-71

65 mixture could reduce androgen signaling during fetal life and induce effects resembling the

66 testicular dysgenesis syndrome (Skakkebaek et al., 2016).

67 To test our hypothesis, and hence contribute new knowledge to the in vivo endocrine modes of

68 action of PBDEs, we conducted a developmental rat toxicity study to determine the effects of

69 early life exposure to DE-71 on endocrine-sensitive endpoints that have not previously been

70 investigated or only investigated at lower doses with smaller statistical power. These endpoints

71 were anogenital distance (AGD) (Schwartz et al., 2019), nipple retention (NR) (Schwartz et al.,

72 2021), and a few selected reproductive postnatal and adult organ weights. We also included

73 analyses of mammary gland development in both male and female offspring by whole-mount

74 assessments. Mammary glands are rarely investigated in reproductive toxicity studies despite

75 their sensitivity to endocrine disruption and importance for reproductive health (Fenton, Reed \&

76 Newbold, 2012; Gouesse et al., 2019). Our study corroborates the endocrine activity of DE-71 in

77 vivo and shows how multiple active endocrine mechanisms can result in a complex in vivo effect

78 pattern. 
81 Materials and Methods

82

83 Chemicals

84 The test compound was a commercial mixture of PBDEs, DE-71 (pentabrominated diphenyl

85 ethers (pentaBDE) from the Great Lakes Chemical Corporation of West Lafayette, IN, CAS No.

86 32534-81-9, lot 7550OK20A), a kind gift from Dr. Kevin Crofton at the U.S. Environmental

87 Protection Agency. The manufacturer reports DE-71 to contain 50-62\% pentaBDE, $24-38 \%$

88 TetraBDE, $4-12 \%$ hexaBDE, and $0-1 \%$ triBDE (amounting to a possible total of 154 congeners)

89 (ENVIRON International Corporation, 2003). Corn oil (Sigma-Aldrich) was used as control

90 compound and vehicle for all treatments.

Animals and treatment

We conducted two rat toxicity studies (Fig. 1). Study 1 (range-finding) aimed at determining the highest doses of DE-71 that could be given to pregnant and lactating rat dams without causing overt systemic toxicity in either dams or offspring. Study 2 (main study) aimed at assessing a series of endocrine sensitive endpoints to clarify the full potential endocrine effect pattern of DE71 in a developmental toxicity study. We also assessed effects on the thyroid hormone system and on neurodevelopment (Ramhøj et al., in prep). In both studies, time-mated nulliparous young adult Wistar rats (HanTac: WH, SPF, Taconic Europe, Ejby, Denmark) were received on gestation day (GD) 3 of pregnancy (day of plug-detection designated GD1), randomly distributed

101 for pairwise housing and on GD4 pseudo-randomly divided into groups with similar weight 102 distribution. 
103 The expected day of delivery, GD23, was designated PD1 irrespective of the actual day of 104 delivery.

105 In Study 1, dams were divided into 4 groups $(n=10$ per group) and exposed to vehicle control or 10620,40 or $60 \mathrm{mg} / \mathrm{kg}$ body weight (bw)/day DE-71. Study 2 was performed in two balanced blocks 107 with 3 groups $(n=22$ per group, in order to have sufficient sample size for mammary gland 108 examinations) exposed to vehicle, 40 or $60 \mathrm{mg} / \mathrm{kg}$ bw/day DE-71. Dosing of the dams was 109 performed daily by gavage in the morning from GD7 to GD22, and again from postnatal day 110 (PD)2 - PD14 in Study 1 (study termination) and PD2-16 in Study 2. Dosing was performed 111 with a metal gastric tube with either vehicle (corn oil) or experimental solutions at a constant 112 volume of $2 \mathrm{ml} / \mathrm{kg} / \mathrm{day}$, with the individual doses based on the body weight of the animal prior to 113 dosing.

Dams were housed pairwise until GD17 and individually thereafter. We used semitransparent

116 plastic cages $(15 \times 27 \times 43 \mathrm{~cm})$ with aspen wood hides and with aspen bedding (Tapvei,

117 Gentofte, Denmark) placed on racks (balanced for treatment group) in an animal room with 118 controlled environmental conditions: reversed light/dark cycles of 12 hours (light from 9 pm-9 119 am, light intensity $500 \mathrm{lux}$ ), temperature $22 \pm 1^{\circ} \mathrm{C}$, humidity $55 \pm 5 \%$, and ventilation changing air 12010 times per hour. All animals were fed ad libitum on a standard diet, Altromin 1314 (soy- and 121 alfalfa-free; Altromin GMbH, Lage, Germany), and acidified tap water was provided ad libitum 122 in PSU bottles (84-ACBT0702SU Techniplast).

123 All endpoint examination procedures were carried out with treatment groups represented in a 124 random order (i.e. not examining all controls first, then treated animals). Examinations and 
125 outcome measures of live animals were conducted blinded to exposure group while data analysis 126 was conducted unblinded.

127 Animals were observed twice daily for health and signs of overt toxicity (such as hunched 128 posture and raised fur). Criteria followed for euthanasia of animals: if it was considered 129 irresponsible or unethical to let the animal live or if they were in a severe condition (expected to 130 die within 24hrs). Accordingly, some litters and pups in Study 1 (see Table 1) were euthanized, 131 as the dams did not take care of them and they were without milk in their stomachs. This

132 phenomenon only took place in Study 1 and was unrelated to exposure, it also includes some 133 dams that cannibalized their pups. In Study 2, one dam was euthanized on GD23 as she appeared 134 unwell and incapable of giving birth (necropsy did not reveal any explanations for the dystocia). Animal experiments were carried out at the DTU National Food Institute (Mørkhøj, Denmark) 136 facilities. Ethical approval was obtained from the Danish Animal Experiments Inspectorate, with 137 authorization number 2012-15-2934-00089 C4. The experiments were overseen by the National 138 Food Institute's in-house Animal Welfare Committee for animal care and use. All methods in the study were performed in accordance with relevant guidelines and regulations.

Birth and postnatal development

142 On the morning after overnight birth, dam and pup body weights were registered, and the pups

143 were sexed and checked for macroscopic anomalies. Body weights of offspring were recorded on 144 PD6, PD10 and PD14 in Study 1 and on PD6, PD14 and PD27 in Study 2.

146 Endocrine sensitive endpoints were investigated in Study 2. Anogenital distance (AGD), the 147 distance between the anus and the genital papilla was measured in all live offspring on PD1, 
148 using a stereomicroscope with a micrometer eyepiece. The AGD index (AGDi) was calculated 149 by dividing the AGD with the cube root of the body weight. On PD14 all offspring had their 150 areolas/nipples counted. Nipple retention (NR) of male pups was defined as the number of 151 areolas/nipples (a dark focal area with or without a nipple bud) visible where nipples are usually 152 located (along the milk lines) in female pups (Schwartz et al., 2021). Both AGD and NR were 153 assessed by the same technician, blinded to exposure group.

154

155 On PD27, one male pup from each litter was weaned and housed pairwise with another male 156 from the same group (when no other males were available, housing was with a littermate).

Necropsy

159

160

161

162

163

164

165

166 168

167 All dams not giving birth (i.e. with resorbed embryos or never pregnant) were humanely killed

Before necropsy was performed, all animals were weighed, anesthetized with $\mathrm{CO}_{2} / \mathrm{O}_{2}$ and killed by decapitation. Selected reproductive organs were examined in offspring killed on PD16, PD27 and PD300 in Study 2. On PD16 one male and one female from each litter were killed and ovaries and ventral prostate were excised and weighed. Mammary glands were collected as whole-mounts from one male and one female on PD27. On PD300, one male offspring from each litter was terminated and prostate together with seminal vesicle was excised and weighed. The ventral prostate was subsequently dissected and weighed alone. on PD3, those with litters were humanely killed, along with remaining pups, on PD14 in Study 1 
169 and on PD27 in Study 2. Implantation scars in uteri were counted to determine pregnancy rates

170 and resorptions.

\section{Whole-mounts}

173 Mammary glands were excised and spread onto a glass slide, covered with parafilm and placed

174 under pressure for 2 hours. The whole-mounts were subsequently fixed in $4 \%$ formalin buffer,

175 stained with alum carmine, dehydrated in alcohol and cleared with xylene. The slides were

176 scanned in a flatbed scanner at 4800 dpi and images of the $4^{\text {th }}$ gland evaluated as described in

177 (Mandrup et al., 2015) for outer area (defined as the smallest polygon enclosing the gland),

178 transverse growth (as defined by (Mandrup et al., 2012)), longitudinal growth, distance to lymph

179 node, distance to $5^{\text {th }}$ gland $\left(n=13-17\right.$, except distance to the $5^{\text {th }}$ gland where $\mathrm{n}=11,4$ and 10 in

180 control, PBDE-40 and PBDE-60, respectively) and number of terminal end buds (TEB) (TEB

181 was defined as tear-drop shaped buds with a diameter $>100 \mu \mathrm{m}$ in zone $\mathrm{C}$, as defined by (Russo

182 \& Russo, 1996) $(\mathrm{n}=17,11$ and 14 in control, PBDE-40 and PBDE-60, respectively). Distance to

183 lymph node was measured and scored (scores 1-3, with a score of 1 representing mammary

184 tissue not reaching the lymph node, score 2 representing mammary tissue reaching the lymph

185 node and score 3 was given to mammary glands where the tissue reaches beyond the lymph

186 node). All data were assessed for males and females separately and blinded to exposure. In

187 addition, for area measurements, longitudinal and transverse growth data from both sexes were

188 pooled for analysis with increased statistical power. Images were analyzed with Image Pro Plus

1897.0 (Media Cybernetics, Bethesda, MD, USA) and calibration performed for each picture before 190 measurements were made. 
193 The alpha level for statistical significance was always set to $5 \%$ and all samples were included in

194 the analysis. Data with normal distribution and homogeneity of variance were analyzed for

195 treatment-related effect differences relative to the control by analysis of variance (ANOVA)

196 followed by Dunnet's correction for multiple comparisons. Data were transformed if these

197 conditions were not met and data not fulfilling the criteria were analyzed using a non-parametric

198 statistical test (Kruskal-Wallis with Dunn's multiple comparison test). When relevant, body

199 weight was included as a covariate in the analysis (ANCOVA), e.g. for terminal organ weights

200 and whole-mount measurements. Litter effects were accounted for by only analyzing one pup per

201 litter, using litter means (offspring growth) or by including the litter as a random effect variable (

202 AGD, NR and pooled whole mounts). Distance to lymph node was evaluated as the distance and

203 as scores. The scores were analyzed using Kruskal-Wallis and using a two-sided Fishers exact

204 test to compare the number of animals with glands reaching past the lymph node with the

205 number not reaching past. The number of nipples/areolas (NR) was assumed to follow a

206 binomial-distribution with a response range between 0 and 12 (12 assumed to reflect the

207 biologically possible maximal number of nipples in rats). Litter effects on NR and over-

208 dispersion in the data were accounted for by using Generalized Estimating Equations (GEE) as

209 reported in (Christiansen et al., 2012).

210 SAS Enterprise guide 4.3 (2010) (SAS Institute Inc, Cary, NC, USA) and GraphPad Prism 5

211 (Graphpad Software, San Diego, CA; USA) was used for statistical analysis. 


\section{Results}

214 Effects on pregnancy, postnatal growth and general toxicity

215 We administered DE-71 at doses of 20,40 and $60 \mathrm{mg} / \mathrm{kg}$ to the pregnant and lactating dams from 216 GD7 to PD14/16. Pregnancy and litter data from Study 1 and Study 2 are listed in Table 1 and

217 Table 2. In Study 1, there were signs of decreased maternal weight gain at 40 and $60 \mathrm{mg} / \mathrm{kg}$.

218 Pups from the $60 \mathrm{mg} / \mathrm{kg}$ bw/day exposure group weighed $8 \%$ less than control pups at birth and

219 gained $20 \%$ less during the first postnatal week. However, none of these findings were

220 statistically significant. Thus, $60 \mathrm{mg} / \mathrm{kg}$ was chosen as the highest dose in Study 2 as it did not

221 induce excessive systemic toxicity in dams and pups.

222 In Study 2, dam weight gain during pregnancy was significantly decreased at $60 \mathrm{mg} / \mathrm{kg}$ (Table

223 2). The high dose pups had decreased body weight on PD6 (Table 2) and body weight remained

224 non-significant reduced throughout the postnatal period (Table 2) with non-significant reductions

225 also on PD27 ( $7 \%$ reduction). Gestation lengths, litter size and pup mortality was not affected

226 by exposure.

Effects on markers of early-life endocrine disruption

A shorter anogenital distance (AGD) and retained nipples in male offspring are considered

230 sensitive markers of disrupted androgen action during development (Schwartz et al., 2019). DE-

23171 exposure induced a significantly shorter AGD and smaller AGDindex (AGDi, AGD adjusted

232 for pup body size) at both 40 and $60 \mathrm{mg} / \mathrm{kg}$. However, the dose-response curve was 'flat' in that

233 reductions in AGD/AGDi were comparable between the two dose groups $(10 / 11 \%$ reduced in the

234 low dose group and 8/6\% in the high dose group). The effect on AGD was also comparable 
235 between both sexes (Fig. 2A and Table 2). Nipple retention was increased in males from the high

236 dose group $(p=0.0249)$. Notably, the mean number of 0.25 nipple in these high dose males is

237 considered very small (Fig. 2B) and falls well within the range of our historic control data

238 (Schwartz et al 2021, in submission - ref to come).

Early-and late-life effects on reproductive tissue weights

241 Weights of selected reproductive organs were assessed both early in life and in adulthood. DE-71

242 at a dose of $60 \mathrm{mg} / \mathrm{kg}$ reduced absolute and relative male PD16 prostate weights with $37 \%$ and

$24342 \%$, respectively (Fig. 3 and Table 3). In female offspring, ovary weights were assessed on

244 PD16 and showed no significant differences between controls and exposed animals.

245 On PD300, changes to prostate weights in exposed offspring were no longer apparent, nor were 246 effects on seminal vesicle or ventral prostate weights (Table 3).

Effects on mammary gland development

249 We assessed mammary gland development by whole-mounts in both male and female offspring

250 at PD27 and found that mammary gland outgrowth was stunted by perinatal exposure to DE-71.

251 In male offspring, the transverse growth and mammary gland areas were statistically

252 significantly reduced in the highest exposure group (Fig. 4). Females appeared to display a

253 similar trend, albeit not statistically significant. When analyzing pooled data from both sexes

254 (litter effects were accounted for by including the litter as a random nested factor in the statistical 255 analysis) there was an effect on area in both dose groups and on the transverse growth in the high 256 dose group (Fig. 4). The observed effects are likely not due to reduced body weight of the 
257 offspring in the high dose as the effects were observed both on absolute numbers and when

258 accounting for the body weight of the animals in the statistical analysis.

259

260 Discussion

261 While production and use of DE-71 is no longer permitted, the constituents of the DE-71 mixture

262 still account for a significant proportion of the brominated flame retardants found in house dust

263 (Bramwell et al., 2016). Humans, including babies and toddlers, continue to be exposed to

264 PBDEs (Klinčić et al., 2020), especially from indoor environments, so a continued focus on the

265 potential detrimental health effects of this exposure is warranted. In this study, we have shown

266 strong endocrine disrupting effects following developmental exposure to DE-71. In contrast to

267 what was expected from existing studies, however, the endocrine disrupting effects seems to be

268 caused by a complex pattern of modalities and not simply anti-androgenic, as discussed in the

269 following.

271 Studies on DE-71, including a Hershberger assay, have clearly shown dose-dependent anti-

272 androgenic effects in male rats when exposure occurs during puberty and adulthood. Effects

273 include delayed preputial separation and reduced prostate weights in animals exposed to 30

$274 \mathrm{mg} / \mathrm{kg}$ and above. Additional reproductive tissues such as seminal vesicle, glandula

275 bulbourethralis and penis showed dose-dependent weight reductions at higher doses (up to 240

$276 \mathrm{mg} / \mathrm{kg} \mathrm{bw} /$ day) (Stoker et al., 2004, 2005). Reductions in reproductive organ weights were seen

277 at $200 \mathrm{mg} / \mathrm{kg}$, but not at $67 \mathrm{mg} / \mathrm{kg}$ or lower in a 28-day exposure study (van der Ven et al., 2008).

278 In contrast, a dietary study using a 70-day exposure to a mixture of PBDEs (of which 52\% was 
279 DE-71), but at maximum dose of $20 \mathrm{mg} / \mathrm{kg}$, found no effects on male reproductive organs,

280 hormone concentrations or sperm count (Ernest et al., 2012). Thus, PBDEs can seemingly elicit

281 anti-androgenic effects in pubertal and adult male rats at doses of $30 \mathrm{mg} / \mathrm{kg}$ or higher. With

282 respect to fetal and early postnatal exposure, however, there are not much available data.

283 One study examines effects on anti-androgenic endpoints after developmental exposure (GD21-

284 PND22) to 1, 10 and $30 \mathrm{mg} / \mathrm{kg}$ DE-71 administered by gavage (Kodavanti et al., 2010). Here

285 effects in the male offspring included a statistically significant 1.8-day delay in preputial

286 separation, a 5\% non-significant shortening of AGD, and a 20\% non-significant decrease in

287 testosterone concentration on PND 60. Using a similar experimental setup to Kodavanti et al

288 (2010), but with higher exposure doses, we observed shorter male AGD of around 10\% in both

289 dose groups (40 and $60 \mathrm{mg} / \mathrm{kg}$ ). We also observed a small effect on male nipple retention in the

290 high dose group, with a mean of 0.25 retained nipples - an incidence rate that lies within our

291 historical control data (Schwartz et al., 2021) but is significantly different from the concurrent

292 control. Reduced male AGD is associated with reproductive malformations and reduced sperm

293 count and is usually indicative of an anti-androgenic mode of action (Christiansen et al., 2008;

294 van den Driesche et al., 2011; Thankamony et al., 2016; Schwartz et al., 2019). However, we

295 also observed shorter female AGD to a very similar degree as in the male offspring, which we do

296 not normally see for clear anti-androgenic compounds (Schwartz et al., 2019). Rather, this effect

297 on female AGD suggests additional modalities induced by the DE-71 mixture.

298 We have previously shown that developmental exposure to BPA and butylparaben, which mainly

299 are considered estrogenic compounds, albeit they also have other modes of action, induce some

300 of the same effects as those observed with the DE-71 mixture: moderately shorter AGD without

301 dose response, similar effects on AGD in both sexes, and no or minimal effect on nipple 
302 retention (Christiansen et al., 2014; Boberg et al., 2016). This could indicate that some of the

303 same endocrine mechanisms are targeted by BPA, butylparaben and DE-71. However, our

304 understanding of the underlying mechanisms causing this particular effect pattern in males and

305 the reduced AGD in females remain elusive (Schwartz 2019). Thus, weak anti-androgenic effects

306 and other endocrine mechanisms could be responsible for the PBDE induced moderate effects on

307 AGD and NR. Notably, intrauterine exposure to PBDEs has been associated with reduced AGD

308 (Luan et al., 2019) and increased risk of hypospadias in boys (Poon et al., 2018; Koren et al.,

309 2019). Although hypospadias has been linked to anti-androgenic chemicals in both humans and

310 rodents, it has also been linked to several compounds with known estrogenic potentials, which

311 suggests the involvement of disrupted androgen-estrogen balance as a critical factor in phallus

312 development (Mattiske \& Pask, 2021). Taken together, this could suggest that the DE-71 mixture

313 induce a more complex disruption to steroid hormone homeostasis which can result in a varied

314 effect pattern.

315 The most marked effect observed in the present study was reduction in prostate weights on

316 PD16, which was around 40\% decreased in exposed versus control animals. Again, this effect

317 could be caused by DE-71 having anti-androgenic properties in the postnatal pups as clearly seen

318 in pubertally exposed animals (Stoker 2004, 2005). However, prostate development is also

319 sensitive to estrogen signaling (Gupta, 2000; Prins \& Korach, 2008; OECD, 2018) which could

320 also contribute to the observed effect. Interestingly, neither BPA nor butylparaben exposure

321 affected PD16 prostate weights in our studies (Boberg 2016, Christiansen 2014) which could

322 indicate that DE-71 works through a different mechanism or possess different ADME

323 (absorption, distribution, metabolism, excretion) properties than BPA and butylparaben in the 
324 postnatal period. Overall, it remains possible that the reduced prostate weight induced by DE-71

325 could have been a result of disrupted androgen-estrogen balance similarly to external genitalia.

326 In addition to the more commonly assessed effect endpoints AGD, NR and prostate weights, we

327 also assessed mammary gland development in both male and female offspring. DE-71 exposure

328 decreased mammary gland area and transverse growth in perinatally exposed offspring,

329 corroborating findings from a DE-71 toxicity study in Long-Evans rats. In the Long-Evans study

330 only female pups were examined, but perinatally exposed female pups showed less outgrowth,

331 fewer lateral branches and limited terminal end bud (TEB) formation on PND 21 (Kodavanti et

332 al., 2010).

333 The delayed mammary gland development in DE-71 exposed offspring is in contrasts to what

334 would be expected for estrogenic compounds, as accelerated or increased mammary gland

335 growth is typically seen after exposure to estrogenic compounds (Mandrup et al., 2012, 2015;

336 Macon \& Fenton, 2013) including for BPA and butylparaben (Boberg et al., 2016; Mandrup et

337 al., 2016). Thus the effects of DE-71 on the mammary glands do not appear to support an

338 estrogenic mode of action. The reduced mammary gland outgrowth caused by DE-71 is also not

339 indicative of anti-androgenic mode of action, as such compounds may not induce an effect on

340 mammary growth in prepubertal stages, but only later in life (Škarda, 2003; Peters et al., 2011;

341 Jacobsen et al., 2012; Mandrup et al., 2015). In fact, the observed reductions in mammary gland

342 outgrowth in the present study may best be attributed to an anti-estrogenic mode of action. This

343 fits well with other studies where anti-estrogenic compounds such as ICI 182,780 can cause

344 decreased mammary gland growth and branching in prepubertal female offspring (Silberstein et 345 al., 1994; Cotroneo, 2002). 
346 Although mammary gland development is sensitive to estrogen signaling, it is also regulated

347 through other signaling pathways. For instance, 2,3,7,8-Tetrachlorodibenzo-p-dioxin (TCDD)

348 given on GD15 can stunt mammary gland growth from PD 4 and persist until PD 68 when the

349 glands still retained undifferentiated terminal structures (Fenton et al., 2002). This effect has

350 been suggested to be mediated through AhR activation (Hushka, Williams \& Greenlee, 1998;

351 Fenton et al., 2002; Helle et al., 2016), a mechanism that may also be relevant for DE-71

352 (Hamers et al., 2006). Also 3,3',4,4',5-pentachlorobiphenyl (PCB-126), which can bind AhR and

353 have anti-estrogenic properties has affected mammary glands similarly to DE-71 (Fenton et al., 354 2002; Muto et al., 2002). Thus various environmental chemicals, including DE-71, seem to cause

355 similar effect patterns on the mammary gland albeit the exact mechanism(s) are still unclear.

In conclusion, we have shown that developmental exposure to DE-71 induces endocrine disrupting effects in rats. We found effects on NR and prostate weights in the male offspring, and effects on AGD and mammary gland development in both males and females. The effect pattern is complex and likely involve various mechanisms of action, which are difficult to pin down. The effect-pattern observed in this study suggest that the mixture acts by anti-androgenic, antiestrogenic and maybe estrogenic modes of action, possibly in combination with effects on other signaling pathways such as AhR signaling. DE-71 exposure causes endocrine disruption in both male and female rat offspring and thus raises concerns for the long-term consequences of human exposure to PBDEs, especially since associations between PBDE concentrations, AGD, and

367 hypospadias in boys have been reported. 
369 Funding

370 This work was funded by the Danish Environmental Protection Agency, Ministry of

371 Environment and Food of Denmark. The funders did not have any influence on design, analysis

372 or reporting of the studies.

\section{Acknowledgments}

375 We would like to thank Mette Voigt Jessen, Heidi Broksø Letting, Lillian Sztuk, Dorte

376 Lykkegaard Korsbech, Ulla El-Baroudy and Sarah Grundt Simonsen for invaluable technical

377 assistance. We also thank Anne Ørngren and staff for animal care and husbandry.

\section{Declaration of interests}

We declare that we have no conflicts of interest.

\section{References}

Attina TM, Hauser R, Sathyanarayana S, Hunt PA, Bourguignon JP, Myers JP, DiGangi J, population-based disease burden and cost analysis. The Lancet Diabetes and Endocrinology 4:996-1003. DOI: 10.1016/S2213-8587(16)30275-3.

Boas M, Feldt-Rasmussen U, Main KM. 2012. Thyroid effects of endocrine disrupting 
401

402

403

404

405

406

407

408

409

410

411

412

413

414

415

416

417

418

419

420

421

422

423

424

425

426

427

428

chemicals. Molecular and cellular endocrinology 355:240-8. DOI: 10.1016/j.mce.2011.09.005.

Boberg J, Axelstad M, Svingen T, Mandrup K, Christiansen S, Vinggaard AM, Hass U. 2016. Multiple endocrine disrupting effects in rats perinatally exposed to butylparaben. Toxicological Sciences 152:244-256. DOI: 10.1093/toxsci/kfw079.

Bramwell L, Glinianaia S V., Rankin J, Rose M, Fernandes A, Harrad S, Pless-Mulolli T. 2016. Associations between human exposure to polybrominated diphenyl ether flame retardants via diet and indoor dust, and internal dose: A systematic review. Environment International 92-93:680-694. DOI: 10.1016/j.envint.2016.02.017.

Christiansen S, Axelstad M, Boberg J, Vinggaard AM, Pedersen GA, Hass U. 2014. Low-dose effects of Bisphenol a on early sexual development in male and female rats. Reproduction 147:477-487. DOI: 10.1530/REP-13-0377.

Christiansen S, Kortenkamp A, Axelstad M, Boberg J, Scholze M, Jacobsen PR, Faust M, Lichtensteiger W, Schlumpf M, Burdorf A, Hass U. 2012. Mixtures of endocrine disrupting contaminants modelled on human high end exposures: An exploratory study in rats. International Journal of Andrology 35:303-316. DOI: 10.1111/j.1365-2605.2011.01242.x.

Christiansen S, Scholze M, Axelstad M, Boberg J, Kortenkamp A, Hass U. 2008. Combined exposure to anti-androgens causes markedly increased frequencies of hypospadias in the rat. International journal of andrology 31:241-248. DOI: 10.1111/j.1365-2605.2008.00866.x.

Cotroneo MS. 2002. Genistein action in the prepubertal mammary gland in a chemoprevention model. Carcinogenesis 23:1467-1474. DOI: 10.1093/carcin/23.9.1467.

van den Driesche S, Scott HM, MacLeod DJ, Fisken M, Walker M, Sharpe RM. 2011. Relative importance of prenatal and postnatal androgen action in determining growth of the penis and anogenital distance in the rat before, during and after puberty. International Journal of Andrology 34. DOI: 10.1111/j.1365-2605.2011.01175.x.

ENVIRON International Corporation. 2003. Voluntary Children's Chemical Evaluation Program (VCCEP). Tier 1 Assessment of the Potential Health Risks to Children Associated With Exposure to the Commercial Pentabromodiphenyl Ether Product. West Lafayette, Indiana, USA.

Ernest SR, Wade MG, Lalancette C, Ma YQ, Berger RG, Robaire B, Hales BF. 2012. Effects of chronic exposure to an environmentally relevant mixture of brominated flame retardants on the reproductive and thyroid system in adult male rats. Toxicological Sciences 127:496507. DOI: $10.1093 /$ toxsci/kfs098.

Fenton SE, Hamm JT, Birnbaum LS, Youngblood GL. 2002. Persistent abnormalities in the rat mammary gland following gestational and lactational exposure to $2,3,7,8$ tetrachlorodibenzo-p-dioxin (TCDD). Toxicological Sciences 67:63-74. DOI: 10.1093/toxsci/67.1.63.

Fenton SE, Reed C, Newbold RR. 2012. Perinatal Environmental Exposures Affect Mammary Development, Function, and Cancer Risk in Adulthood. Annual Review of Pharmacology and Toxicology 52:455-479. DOI: 10.1146/annurev-pharmtox-010611-134659. 
429

430

431

432

433

434

435

436

437

438

439

440

441

442

443

444

445

446

447

448

449

450

451

452

453

454

455

456

457

458

459

460

461

462

463

464

465

466

467

468

469

Gore AC, Chappell VA, Fenton SE, Flaws JA, Nadal A, Prins GS, Toppari J, Zoeller RT. 2015. EDC-2: The Endocrine Society's second Scientific Statement on endocrine-disrupting chemicals. Endocrine Reviews 36:E1-E150. DOI: 10.1210/er.2015-1093.

Gouesse R-J, Lavoie M, Dianati E, Wade MG, Hales BF, Robaire B, Plante I. 2019. Gestational and Lactational Exposure to an Environmentally Relevant Mixture of Brominated Flame Retardants Downregulates Junctional Proteins, Thyroid Hormone Receptor $\alpha 1$ Expression, and the Proliferation-Apoptosis Balance in Mammary Glands Post Puberty. Toxicological Sciences 171:13-31. DOI: 10.1093/toxsci/kfz147.

Gupta C. 2000. The role of estrogen receptor, androgen receptor and growth factors diethylstilbestrol-induced programming of prostate differentiation. Urological Research 28:223-229. DOI: 10.1007/s002400000107.

Hamers T, Kamstra JH, Sonneveld E, Murk AJ, Kester MH a, Andersson PL, Legler J, Brouwer A. 2006. In vitro profiling of the endocrine-disrupting potency of brominated flame retardants. Toxicological sciences : an official journal of the Society of Toxicology 92:15773. DOI: $10.1093 /$ toxsci/kfj 187.

Helle J, Bader MI, Keiler AM, Zierau O, Vollmer G, Chittur S V., Tenniswood M, Kretzschmar G. 2016. Cross-Talk in the female rat mammary gland: Influence of aryl hydrocarbon receptor on estrogen receptor signaling. Environmental Health Perspectives 124:601-610. DOI: $10.1289 /$ ehp. 1509680.

Hushka LJ, Williams JS, Greenlee WF. 1998. Characterization of 2,3,7,8tetrachlorodibenzofuran-dependent suppression and AH receptor pathway gene expression in the developing mouse mammary gland. Toxicology and Applied Pharmacology 152:200210. DOI: $10.1006 /$ taap. 1998.8508 .

Jacobsen PR, Axelstad M, Boberg J, Isling LK, Christiansen S, Mandrup KR, Berthelsen LO, Vinggaard AM, Hass U. 2012. Persistent developmental toxicity in rat offspring after low dose exposure to a mixture of endocrine disrupting pesticides. Reproductive Toxicology 34:237-250.

Klinčić D, Dvoršćak M, Jagić K, Mendaš G, Herceg Romanić S. 2020. Levels and distribution of polybrominated diphenyl ethers in humans and environmental compartments: a comprehensive review of the last five years of research. Environmental Science and Pollution Research 27:5744-5758. DOI: 10.1007/s11356-020-07598-7.

Kodavanti PRS, Coburn CG, Moser VC, MacPhail RC, Fenton SE, Stoker TE, Rayner JL, Kannan K, Birnbaum LS. 2010. Developmental exposure to a commercial PBDE Mixture, DE-71: Neurobehavioral, hormonal, and reproductive effects. Toxicological Sciences 116:297-312. DOI: 10.1093/toxsci/kfq105.

Koren G, Carnevale A, Ling J, Ozsarfati J, Kapur B, Bagli D. 2019. Fetal exposure to polybrominated diphenyl ethers and the risk of hypospadias: focus on the congeners involved. Journal of Pediatric Urology 15:405.e1-405.e6. DOI: 10.1016/j.jpurol.2019.05.023.

Luan M, Liang H, Yang F, Yuan W, Chen A, Liu X, Ji H, Wen S, Miao M. 2019. Prenatal polybrominated diphenyl ethers exposure and anogenital distance in boys from a Shanghai 
492

493

494

495

496

497

498

499

500

501

502

503

504

505

506

507

birth cohort. International Journal of Hygiene and Environmental Health 222:513-523. DOI: 10.1016/j.ijheh.2019.01.008.

Macon MB, Fenton SE. 2013. Endocrine disruptors and the breast: Early life effects and later life disease. Journal of Mammary Gland Biology and Neoplasia 18:43-61. DOI: 10.1007/s10911-013-9275-7.

Mandrup K, Boberg J, Isling LK, Christiansen S, Hass U. 2016. Low-dose effects of bisphenol A on mammary gland development in rats. Andrology 4:673-683. DOI: 10.1111/andr.12193.

Mandrup KR, Hass U, Christiansen S, Boberg J. 2012. Perinatal ethinyl oestradiol alters mammary gland development in male and female Wistar rats. International Journal of Andrology 35:385-396. DOI: 10.1111/j.1365-2605.2012.01258.x.

Mandrup KR, Johansson HKL, Boberg J, Pedersen AS, Mortensen MS, Jørgensen JS, Vinggaard AM, Hass U. 2015. Mixtures of environmentally relevant endocrine disrupting chemicals affect mammary gland development in female and male rats. Reproductive Toxicology 54:47-57. DOI: 10.1016/j.reprotox.2014.09.016.

Mattiske DM, Pask AJ. 2021. Endocrine disrupting chemicals in the pathogenesis of hypospadias; developmental and toxicological perspectives. Current Research in Toxicology 2:179-191. DOI: 10.1016/j.crtox.2021.03.004.

Mercado-Feliciano M, Bigsby RM. 2008a. Hydroxylated metabolites of the polybrominated diphenyl ether mixture DE-71 Are weak estrogen receptor- $\alpha$ ligands. Environmental Health Perspectives 116:1315-1321. DOI: 10.1289/ehp.11343.

Mercado-Feliciano M, Bigsby RM. 2008b. The Polybrominated diphenyl ether mixture DE-71 1s. Mildly estrogenic. Environmental Health Perspectives 116:605-611. DOI: 10.1289/ehp.10643.

Muto T, Wakui S, Imano N, Nakaaki K, Takahashi H, Hano H, Furusato M, Masaoka T. 2002. Mammary gland differentiation in female rats after prenatal exposure to $3,3^{\prime}, 4,4^{\prime}, 5-$ pentachlorobiphenyl. Toxicology 177:197-205. DOI: 10.1016/S0300-483X(02)00224-X.

OECD. 2018. Revised Guidance Document 150 on Standardised Test Guidelines for Evaluating Chemicals for Endocrine Disruption. OECD Series on Testing and Assessment.

Peters AA, Ingman W V., Tilley WD, Butler LM. 2011. Differential effects of exogenous androgen and an androgen receptor antagonist in the peri- and postpubertal murine mammary gland. Endocrinology 152:3728-3737. DOI: 10.1210/en.2011-1133.

Poon S, Koren G, Carnevale A, Aleksa K, Ling J, Ozsarfati J, Kapur BM, Bagli D. 2018. Association of In Utero Exposure to Polybrominated Diphenyl Ethers With the Risk of Hypospadias. JAMA Pediatrics 172:851-856. DOI: 10.1001/jamapediatrics.2018.1492.

Prins GS, Korach KS. 2008. The role of estrogens and estrogen receptors in normal prostate growth and disease. Steroids 73:233-244. DOI: 10.1016/j.steroids.2007.10.013.

Russo IH, Russo J. 1996. Mammary gland neoplasia in long-term rodent studies. Environmental Health Perspectives 104:938-967. DOI: 10.1289/ehp.96104938. 
508

509

510

511

512

513

514

515

516

517

518

519

520

521

522

523

524

525

526

527

528

529

530

531

532

533

534

535

536

537

538

539

540

541

542

543

544

545

546

547

Schwartz CL, Christiansen S, Hass U, Ramhøj L, Löbl NM, Svingen T. 2021. On the use and interpretation of areola/nipple retention as a biomarker for anti-androgenic effects in rat toxicity studies. Frontiers in Toxicology TBA:TBA. DOI: 10.3389/ftox.2021.730752.

Schwartz CL, Christiansen S, Vinggaard AM, Axelstad M, Hass U, Svingen T. 2019. Anogenital distance as a toxicological or clinical marker for fetal androgen action and risk for reproductive disorders. Archives of Toxicology 93:253-272. DOI: 10.1007/s00204-0182350-5.

Silberstein GB, Horn K van, Shyamala G, Daniel CW. 1994. Essential role of endogenous estrogen in directly stimulating mammary growth demonstrated by implants containing pure antiestrogens. Endocrinology 134:84-90.

Skakkebaek NE, Rajpert-De Meyts E, Buck Louis GM, Toppari J, Andersson AM, Eisenberg ML, Jensen TK, Jørgensen N, Swan SH, Sapra KJ, Ziebe S, Priskorn L, Juul A. 2016. Male reproductive disorders and fertility trends: Influences of environment and genetic susceptibility. Physiological Reviews 96:55-97. DOI: 10.1152/physrev.00017.2015.

Škarda J. 2003. Bioassay of steroid hormone agonist and antagonist activities of anti-androgens on mammary gland, seminal vesicles and spleen of male mice. Journal of Veterinary Medicine Series A: Physiology Pathology Clinical Medicine 50:204-212. DOI: 10.1046/j.1439-0442.2003.00526.x.

Stockholm Convention. 2019.Stockholm Convention. Available at http://chm.pops.int/TheConvention/ThePOPs/AllPOPs/tabid/2509/Default.aspx

Stoker TE, Cooper RL, Lambright CS, Wilson VS, Furr J, Gray LE. 2005. In vivo and in vitro anti-androgenic effects of DE-71, a commercial polybrominated diphenyl ether (PBDE) mixture. Toxicology and applied pharmacology 207:78-88. DOI: 10.1016/j.taap.2005.05.010.

Stoker TE, Laws SC, Crofton KM, Hedge JM, Ferrell JM, Cooper RL. 2004. Assessment of DE71, a commercial polybrominated diphenyl ether (PBDE) mixture, in the EDSP male and female pubertal protocols. Toxicological sciences : an official journal of the Society of Toxicology 78:144-55. DOI: 10.1093/toxsci/kfh029.

Thankamony A, Pasterski V, Ong KK, Acerini CL, Hughes IA. 2016. Anogenital distance as a marker of androgen exposure in humans. Andrology 4:616-625. DOI: 10.1111/andr.12156.

Trasande L, Zoeller RT, Hass U, Kortenkamp A, Grandjean P, Myers JP, DiGangi J, Hunt PM, Rudel R, Sathyanarayana S, Bellanger M, Hauser R, Legler J, Skakkebaek NE, Heindel JJ. 2016. Burden of disease and costs of exposure to endocrine disrupting chemicals in the European Union: an updated analysis. Andrology 4:565-572. DOI: 10.1111/andr.12178.

van der Ven LTM, van de Kuil T, Verhoef A, Leonards PEG, Slob W, Cantón RF, Germer S, Hamers T, Visser TJ, Litens S, Håkansson H, Fery Y, Schrenk D, van den Berg M, Piersma AH, Vos JG. 2008. A 28-day oral dose toxicity study enhanced to detect endocrine effects of a purified technical pentabromodiphenyl ether (pentaBDE) mixture in Wistar rats. Toxicology 245:109-22. DOI: 10.1016/j.tox.2007.12.016.

Zhang T, Zhou X, Xu A, Tian Y, Wang Y, Zhang Y, Gu Q, Wang SQ, Wang Z. 2020. Toxicity

Peer) reviewing PDF | (2021:09:65895:1:0:NEW 24 Nov 2021) 
548 of polybrominated diphenyl ethers (PBDEs) on rodent male reproductive system: A

549 systematic review and meta-analysis of randomized control studies. Science of the Total

$550 \quad$ Environment 720. DOI: 10.1016/j.scitotenv.2020.137419.

551 
552 Figure 1. Study design for developmental toxicity studies with a technical mixture of brominated

553 flame retardants, DE-71. Study $1(\mathrm{n}=10$ dams) was a range-finding study to choose doses for

554 the larger Study 2 ( $n=22$ dams) that investigated endocrine sensitive endpoints in the perinatally

555 exposed rat offspring. AGD: anogenital distance, Bw: body weight, GD: gestation day, NR:

556 nipple retention, PBDE: polybrominated diphenyl ethers (DE-71), PD: postnatal day.

557

558

Figure 2. Anogenital distance (AGD) and nipple retention (NR) in rat offspring after perinatal exposure to brominated flame retardants (DE-71). (A) Reduced AGD in PD1 male and female offspring. (B) NR in male PD14 offspring. $n=19-21$ litters. Statistical analysis performed on all pups from each litter with adjustment for litter effects. Litter means $+\mathrm{SEM},{ }^{*} \mathrm{p}<0.05$ and $* * * \mathrm{p}<0.001$ compared to control.

Figure 3. Relative ventral prostate weights in PD16 male offspring after perinatal exposure to 565 brominated flame retardants (DE-71). Mean+SEM, $\mathrm{n}=16-19 . * * * \mathrm{p}<0.001$.

567 Figure 4. Mammary gland development in PD27 offspring after perinatal exposure to

568 brominated flame retardants (DE-71). (A) Area of the mammary gland was reduced. (B)

569 Transverse growth of the mammary gland was reduced at the highest dose. Mean+SEM. $\mathrm{n}=14$ -

57017 males, $\mathrm{n}=18-20$ females, $\mathrm{n}=32-37$ for males and females pooled and litter effects accounted

571 for in the statistical analysis. Mean+SEM. ${ }^{*} \mathrm{p}<0.05$ (bw as covariate), $\# \mathrm{p}<0.05$ using ANOVA. 


\section{Figure 1}

Study design for developmental toxicity studies with a technical mixture of brominated flame retardants, DE-71.

Study 1 ( $n=10$ dams) was a range-finding study to choose doses for the larger Study 2 ( $n=$ 22 dams) that investigated endocrine sensitive endpoints in the perinatally exposed rat offspring. AGD: anogenital distance, Bw: body weight, GD: gestation day, NR: nipple retention, PBDE: polybrominated diphenyl ethers (DE-71), PD: postnatal day.

\begin{tabular}{|c|c|c|c|c|c|}
\hline \multicolumn{5}{|l|}{ Study design } & Study $\mathbf{1}$, treatment groups, $n=10$ \\
\hline \multirow{2}{*}{\multicolumn{3}{|c|}{ Study 1 - range-finding study }} & \multirow{2}{*}{\multicolumn{2}{|c|}{$\begin{array}{l}\text { Study ends } \\
\text { Litter data }\end{array}$}} & $\begin{array}{l}\text { Control } \\
\text { PBDE - } 20 \mathrm{mg} / \mathrm{kg} \text { bw/day }\end{array}$ \\
\hline & & & & & PBDE - $40 \mathrm{mg} / \mathrm{kg}$ bw/day \\
\hline Exposure of dams & Birth & Bw & & Bw & PBDE - 60 mg/kg bw/day \\
\hline GD7 & $\mathrm{GD} 23=\mathrm{PD} 1$ & PD6 & PD10 & PD14 PD16 & PD300 \\
\hline \multirow[b]{2}{*}{ Study 2 - main study } & $\begin{array}{l}\text { Birth } \\
\text { Bw } \\
\text { AGD }\end{array}$ & Bw & \multirow{2}{*}{\multicolumn{2}{|c|}{$\begin{array}{c}\text { Bw } \\
\text { NR } \\
\text { Bw } \\
\text { Litter data } \\
\text { Ventral prostate } \\
\text { Ovaries }\end{array}$}} & $\begin{array}{cc}\text { Mammary gland } & \text { Prostate+seminal vesicle } \\
\text { whole mounts } & \text { Ventral prostate }\end{array}$ \\
\hline & & & & & $\begin{array}{l}\text { Study } 2 \text {, treatment groups, } n=22 \\
\text { Control } \\
\text { PBDE }-40 \mathrm{mg} / \mathrm{kg} \text { bw/day } \\
\text { PBDE }-60 \mathrm{mg} / \mathrm{kg} \text { bw/day }\end{array}$ \\
\hline
\end{tabular}


Figure 2

Anogenital distance (AGD) and nipple retention (NR) in rat offspring after perinatal exposure to brominated flame retardants (DE-71)

(A) Reduced AGD in PD1 male and female offspring. (B) NR in male PD14 offspring. $n=19-21$ litters. Statistical analysis performed on all pups from each litter with adjustment for litter effects. Litter means+SEM, $* \mathrm{p}<0.05$ and $* * * p<0.001$ compared to control.

A

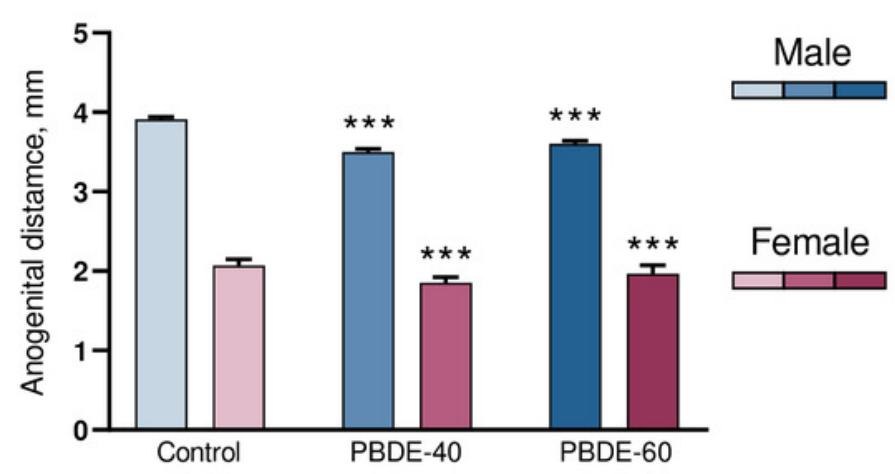

B

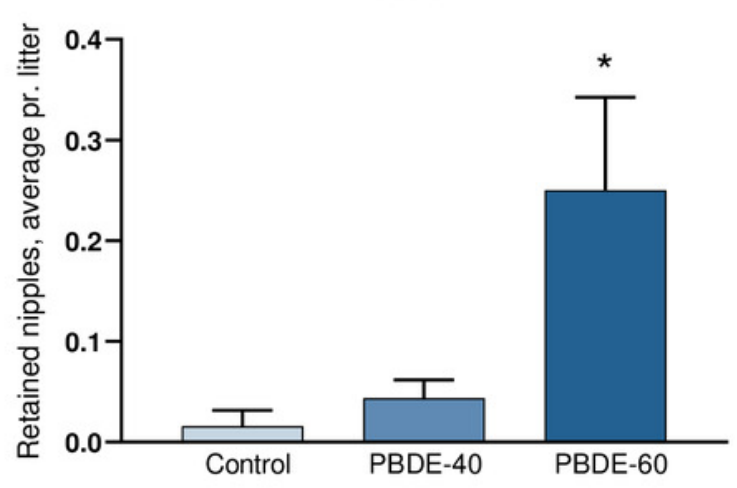


Figure 3

Relative ventral prostate weights in PD16 male offspring after perinatal exposure to brominated flame retardants (DE-71).

Mean+SEM, $\mathrm{n}=16-19 .{ }^{* * *} \mathrm{p}<0.001$.

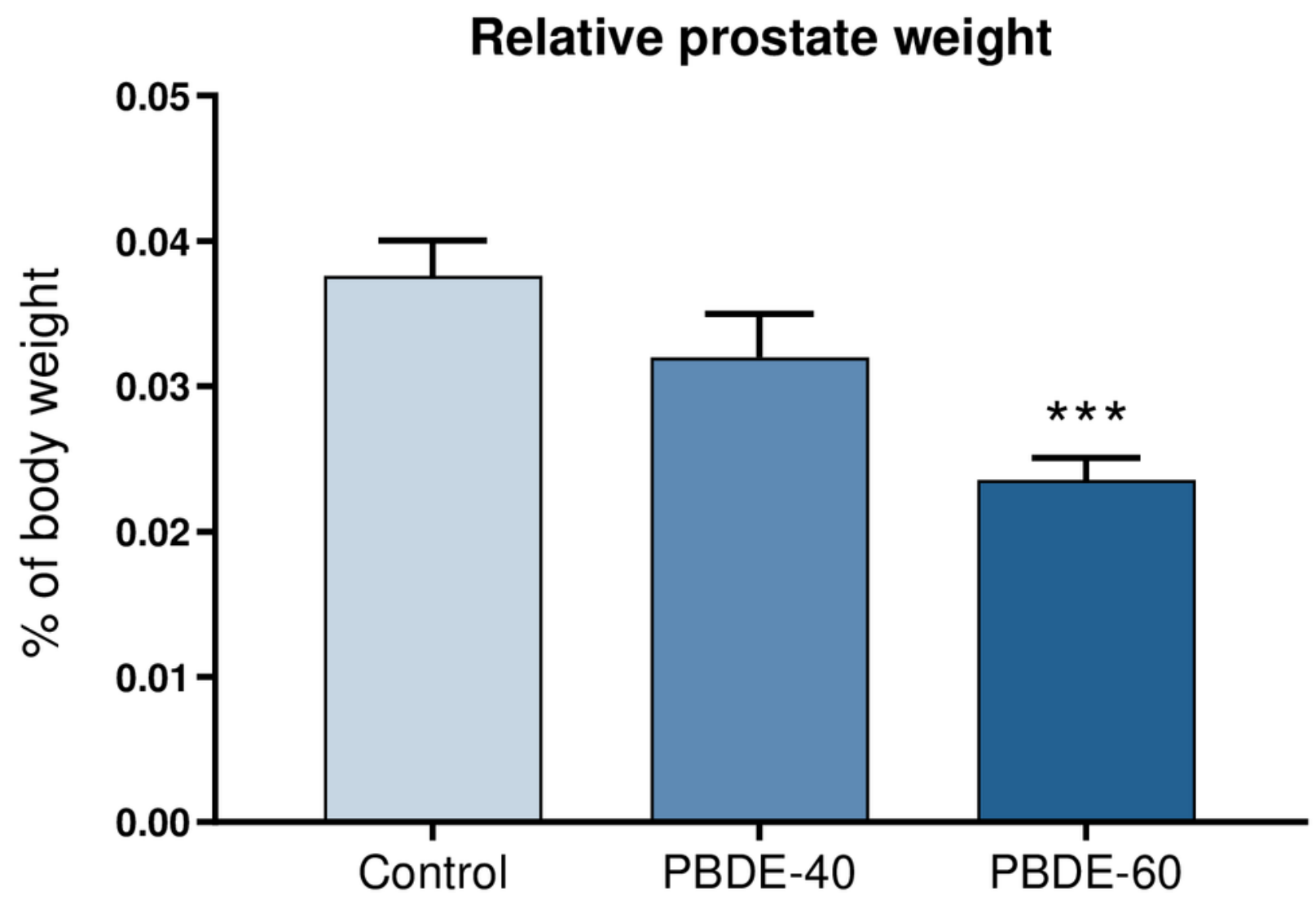


Figure 4

Mammary gland development in PD27 offspring after perinatal exposure to brominated flame retardants (DE-71)

(A) Area of the mammary gland was reduced. (B) Transverse growth of the mammary gland was reduced at the highest dose. Mean+SEM. $n=14-17$ males, $n=18-20$ females, $n=$ 32-37 for males and females pooled and litter effects accounted for in the statistical analysis. Mean+SEM. $* p<0.05$ (bw as covariate), $\# p<0.05$ using ANOVA.

A

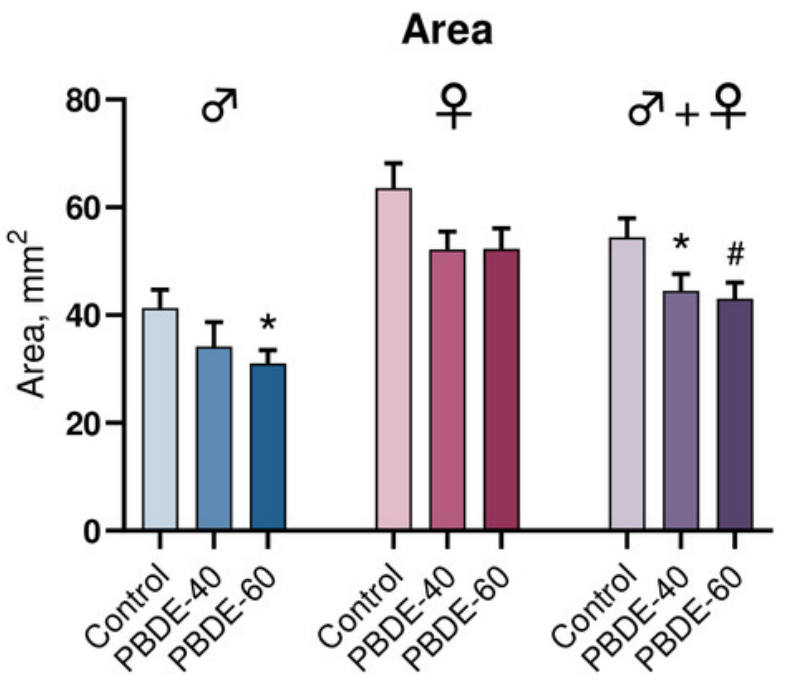

B

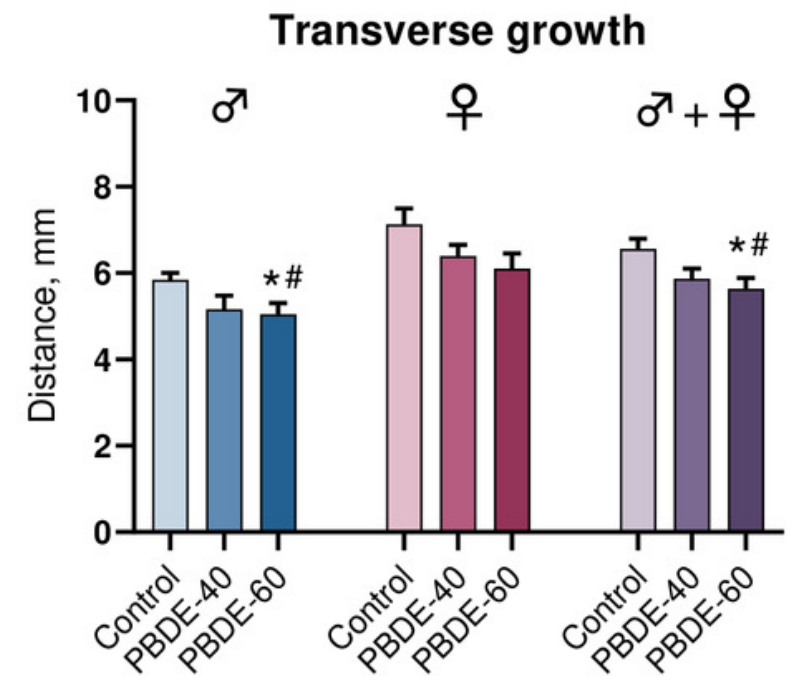




\section{Table 1 (on next page)}

Pregnancy and litter data: Study 1

bw: body weight, GD: Gestation day, PD: postnatal day. ${ }^{a}$ Unrelated to exposure there were dams cannibalizing or not taking care of their pups in this study. Data represented as Mean \pm SD. 
1 Table 1. Pregnancy and litter data: Study 1

\begin{tabular}{|c|c|c|c|c|}
\hline Dams and litters & Control & 20 mg/kg DE-71 & 40 mg/kg DE-71 & $60 \mathrm{mg} / \mathrm{kg} \mathrm{DE}-71$ \\
\hline No. of dams (viable litters) & $\mathrm{n}=10(8)$ & $\mathrm{n}=10(7)$ & $\mathrm{n}=10(8)$ & $\mathrm{n}=10(8)$ \\
\hline \multicolumn{5}{|l|}{ Dam body weight gain } \\
\hline Dam bw-gain, GD7-GD21 (g) & $79.6 \pm 13.0$ & $87.1 \pm 8.4$ & $82.2 \pm 14.6$ & $90 \pm 17.2$ \\
\hline Dam bw-gain, GD7-PD1 (g) & $16.5 \pm 8.8$ & $8.9 \pm 20.0$ & $2.1 \pm 10.9$ & $4.5 \pm 9.2$ \\
\hline Dam bw-gain, PD1-14 (g) & $15.6 \pm 13.4$ & $16.6 \pm 14.3$ & $26.1 \pm 10.2$ & $28.4 \pm 10.8$ \\
\hline \multicolumn{5}{|l|}{ Litters } \\
\hline Gestation length & $22.9 \pm 0.6$ & $23.0 \pm 0.0$ & $23.0 \pm 0.0$ & $23.0 \pm 0.0$ \\
\hline $\begin{array}{l}\text { Postimplantation loss (prenatal } \\
\text { mortality) }(\%)\end{array}$ & $16.0 \pm 12.6$ & $6.0 \pm 5.0$ & $4.7 \pm 5.5$ & $4.9 \pm 6.4$ \\
\hline $\begin{array}{l}\text { Perinatal loss }{ }^{a} \text { (pre- and } \\
\text { postnatal mortality) (\%) }\end{array}$ & $32.3 \pm 37.7$ & $21.0 \pm 32.0$ & $6.6 \pm 6.5$ & $27.6 \pm 39.1$ \\
\hline Litter size (no.) & $9.6 \pm 4.3$ & $11.3 \pm 2.5$ & $11.8 \pm 3.4$ & $11.8 \pm 3.4$ \\
\hline Postnatal deatha (\%) & $21.0 \pm 41.8$ & $15.1 \pm 34.7$ & $2.0 \pm 3.7$ & $23.5 \pm 13.0$ \\
\hline Sex ratio, males / females (\%) & $58 / 42 \pm 17.9$ & $56 / 44 \pm 11.7$ & $46 / 54 \pm 12.9$ & $52 / 48 \pm 9.2$ \\
\hline \multicolumn{5}{|l|}{ Offspring } \\
\hline Male birth weight (g) & $6.2 \pm 0.7$ & $6.1 \pm 0.9$ & $6.2 \pm 0.9$ & $5.8 \pm 0.7$ \\
\hline Female birth weight $(\mathrm{g})$ & $5.9 \pm 0.6$ & $5.9 \pm 0.6$ & $5.9 \pm 0.7$ & $5.5 \pm 0.7$ \\
\hline Mean bw-gain PD1-6 (g) & $7.4 \pm 1.2$ & $6.9 \pm 1.7$ & $6.0 \pm 1.3$ & $5.9 \pm 1.5$ \\
\hline Mean bw-gain PD6-14 (g) & $23.1 \pm 2.0$ & $24.2 \pm 1.0$ & $21.7 \pm 1.3$ & $22.1 \pm 1.8$ \\
\hline
\end{tabular}

2 bw: body weight, GD: Gestation day, PD: postnatal day.

3 a Unrelated to exposure there were dams cannibalizing or not taking care of their pups in this study.

4 Data represented as Mean \pm SD. 


\section{Table 2 (on next page)}

Pregnancy and litter data: Study 2

${ }^{*} \mathrm{p}<0.05 .{ }^{* * *} \mathrm{p}<0.001$. Bw: body weight, GD: Gestation day, PD: postnatal day. Data shown as Mean \pm SD. 
1 Table 2. Pregnancy and litter data: Study 2

\begin{tabular}{|c|c|c|c|}
\hline Dams and litters & Control & 40 mg/kg DE-71 & 60 mg/kg DE-71 \\
\hline No. of dams (viable litters) & $\mathrm{n}=22(21)$ & $\mathrm{n}=22(20)$ & $\mathrm{n}=22(20)$ \\
\hline \multicolumn{4}{|l|}{ Dam body weight gain } \\
\hline Dam, GD7-GD21 (g) & $84.5 \pm 13.6$ & $83.8 \pm 14.2$ & $78.6 \pm 16.6$ \\
\hline Dam, GD7-PD1 (g) & $13.0 \pm 11.1$ & $11.8 \pm 12.5$ & $2.8 \pm 11.0^{*}$ \\
\hline Dam, PD1-14 (g) & $42.4 \pm 13.1$ & $37.0 \pm 11.4$ & $39.9 \pm 17.8$ \\
\hline Dam, PD14-27 (g) & $-20.9 \pm 11.6$ & $-21.5 \pm 9.6$ & $-12.0 \pm 10.9^{*}$ \\
\hline \multicolumn{4}{|l|}{ Litters } \\
\hline Gestation length & $23.0 \pm 0.3$ & $23.0 \pm 0.2$ & $23.0 \pm 0.1$ \\
\hline $\begin{array}{l}\text { Postimplantation loss (prenatal } \\
\text { mortality) (\%) }\end{array}$ & $6.6 \pm 8.6$ & $6.3 \pm 7.9$ & $11.7 \pm 23.9$ \\
\hline $\begin{array}{l}\text { Perinatal loss }{ }^{a} \text { (pre- and } \\
\text { postnatal mortality) (\%) }\end{array}$ & $10.3 \pm 8.3$ & $7.1 \pm 8.0$ & $21.2 \pm 31.9$ \\
\hline Litter size (no.) & $10.9 \pm 3.0$ & $10.7 \pm 3.1$ & $10.8 \pm 3.6$ \\
\hline Postnatal death (\%) & $3.8 \pm 6.1$ & $0.8 \pm 2.5$ & $9.5 \pm 26.0$ \\
\hline Sex ratio, males / females (\%) & $45 / 55 \pm 14$ & $46 / 54 \pm 16$ & $46 / 54 \pm 14$ \\
\hline \multicolumn{4}{|l|}{ Offspring } \\
\hline Male birth weight (g) & $6.2 \pm 0.4$ & $6.3 \pm 0.7$ & $5.9 \pm 0.6$ \\
\hline Female birth weight (g) & $5.9 \pm 0.5$ & $6.0 \pm 0.6$ & $5.8 \pm 0.7$ \\
\hline Male AGDi & $2.13 \pm 0.06$ & $1.90 \pm 0.09^{* * *}$ & $1.99 \pm 0.08^{* * *}$ \\
\hline Female AGDi & $1.15 \pm 0.04$ & $1.02 \pm 0.03^{* * *}$ & $1.09 \pm 0.05^{\star * *}$ \\
\hline Male bw PD6 (g) & $12.5 \pm 1.8$ & $12.3 \pm 2.7$ & $11.1 \pm 2.1^{* *}$ \\
\hline Female bw PD6 (g) & $12.2 \pm 1.9$ & $12.0 \pm 2.3$ & $11.1 \pm 2.3^{\star *}$ \\
\hline Male bw PD14 (g) & $27.9 \pm 4.1$ & $28.1 \pm 5.9$ & $25.6 \pm 4.4$ \\
\hline Female bw PD14 (g) & $27.3 \pm 4.4$ & $27.5 \pm 5.5$ & $25.8 \pm 5.2$ \\
\hline Male bw PD27 (g) & $65.3 \pm 1.7$ & $65.1 \pm 1.9$ & $61.2 \pm 1.6$ \\
\hline Female bw PD27 (g) & $67.2 \pm 1.7$ & $67.5 \pm 2.5$ & $62.0 \pm 1.8$ \\
\hline
\end{tabular}




\section{Table 3(on next page)}

Absolute organ weights after perinatal exposure to a mixture of brominated flame retardants, Study 2

Mean \pm SD. ${ }^{* * *} p<0.001$ compared to control with body weight as covariate in the analysis. 
1 Table 3. Absolute organ weights after perinatal exposure to a mixture of brominated flame retardants,

2 Study 2

\begin{tabular}{|c|c|c|c|}
\hline Offspring & Control & 40 mg/kg DE-71 & $60 \mathrm{mg} / \mathrm{kg} \mathrm{DE-71}$ \\
\hline Male PD16 & $n=19$ & $\mathrm{n}=18$ & $\mathrm{n}=17$ \\
\hline Body weight, g & $30.6 \pm 4.2$ & $29.95 \pm 5.7$ & $28.60 \pm 5.1$ \\
\hline Ventral prostate, mg & $11.8 \pm 4.9$ & $9.8 \pm 5.1$ & $6.8 \pm 2.2^{* \star *}$ \\
\hline Female PD16 & $n=21$ & $n=20$ & $\mathrm{n}=19$ \\
\hline Body weight, g & $30.8 \pm 5.0$ & $31.0 \pm 6.4$ & $29.1 \pm 5.6$ \\
\hline Ovary, right, mg & $2.4 \pm 0.6$ & $2.3 \pm 0.5$ & $2.2 \pm 0.5$ \\
\hline Ovary, left, mg & $2.3 \pm 0.6$ & $2.3 \pm 0.7$ & $2.4 \pm 0.6$ \\
\hline Male PD300 & $n=19$ & $n=18$ & $\mathrm{n}=18$ \\
\hline Body weight, g & $516 \pm 69.5$ & $549 \pm 60.6$ & $529 \pm 55.6$ \\
\hline $\begin{array}{l}\text { Prostate and seminal } \\
\text { vesicle, } \mathrm{g}\end{array}$ & $3.02 \pm 0.38$ & $3.14 \pm 0.37$ & $3.10 \pm 0.35$ \\
\hline Ventral prostate, $\mathrm{g}$ & $0.74 \pm 0.12$ & $0.69 \pm 0.17$ & $0.72 \pm 0.14$ \\
\hline
\end{tabular}

3 Mean \pm SD. $* * * \mathrm{p}<0.001$ compared to control with body weight as covariate in the analysis. 
Figure 5

Graphical abstract

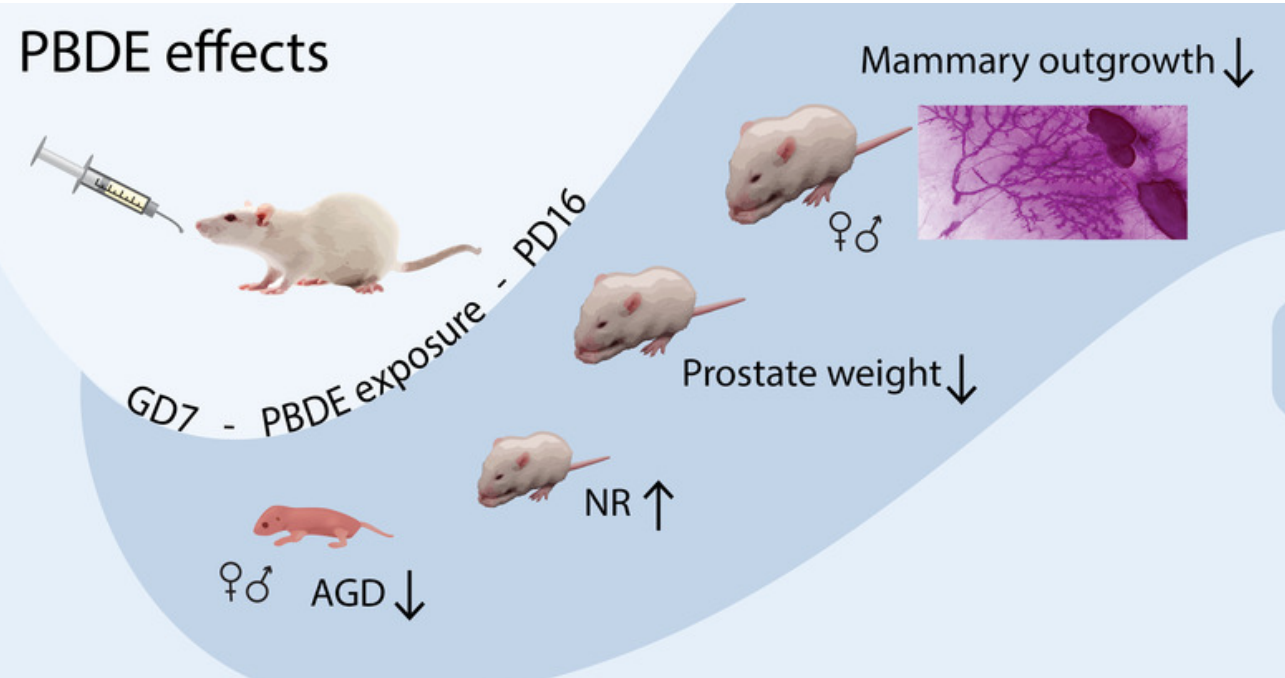

Complex endocrine effect pattern

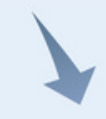

Reproductive health $\downarrow$ 